\title{
Thom polynomials and Schur functions: the singularities $I_{2,2}(-)$
}

\author{
Piotr Pragacz* \\ Institute of Mathematics of Polish Academy of Sciences \\ Śniadeckich 8, 00-956 Warszawa, Poland \\ P.Pragacz@impan.gov.pl
}

(29.03.2006; revised 15.03.2007)

To the memory of Professor Stanistaw Balcerzyk (1932-2005)

\begin{abstract}
We give the Thom polynomials for the singularities $I_{2,2}$ associated with maps $\left(\mathbf{C}^{\bullet}, 0\right) \rightarrow\left(\mathbf{C}^{\bullet+k}, 0\right)$ with parameter $k \geq 0$. Our computations combine the characterization of Thom polynomials via the "method of restriction equations" of Rimanyi et al. with the techniques of Schur functions.
\end{abstract}

\section{Introduction}

The global behavior of singularities is governed by their Thom polynomials (cf. [35, 14, 11, 11], 31]). Knowing the Thom polynomial of a singularity $\eta$, denoted $\mathcal{T}^{\eta}$, one can compute the cohomology class represented by the $\eta$-points of a map. We do not attempt here to survey all activities related to computations of Thom polynomials, which are difficult tasks in general.

In the present paper, following a series of papers by Rimanyi et al. 32, [31, 7], 2], we study the Thom polynomials for the singularities $I_{2,2}$ of the maps $\left(\mathbf{C}^{\bullet}, 0\right) \rightarrow\left(\mathbf{C}^{\bullet+k}, 0\right)$ with parameter $k \geq 0$.

The way of obtaining the thought Thom polynomial is through the solution of a system of linear equations, which is fine when we want to find one concrete Thom polynomial, say, for a fixed $k$. However, if we want to find the Thom polynomials for a series of singularities, associated with maps $\left(\mathbf{C}^{\bullet}, 0\right) \rightarrow\left(\mathbf{C}^{\bullet+k}, 0\right)$ with $k$ as a parameter, we have to solve simultaneously

2000 Mathematics Subject Classification. 05E05, 14N10, 57R45.

Key words and phrases. Thom polynomials, singularities, global singularity theory, classes of degeneracy loci, Schur functions, resultants.

* Research supported by a KBN grant, by TÜBİTAK (during the stay at the METU in Ankara), and by the Humboldt Stiftung (during the stay at the MPIM in Bonn). 
a countable family of systems of linear equations. As stated by Rimanyi in [31, p. 512 :

"However, another challenge is to find Thom polynomials containing $k$ as a parameter."

We do it here for the restriction equations for the singularities $I_{2,2}$ (any $k$ ) with the help of Schur functions. It appears that a use of these functions puts a transparent structure on computations of Thom polynomials. In particular, we get in this way some recursive formulas (cf., e.g., Lemma 14) that are not so easy to find using other bases (e.g. the Chern monomial basis that was used in [31] and in the references quoted there). In fact, various recursions play a prominent role in the present paper - apart from Lemma 14, see Eq. (58). Let us note that in a recent paper [6], Feher and Komuves compute Thom polynomials for some second order Thom-Boardman singularities also using Schur functions, and obtain similar recursions for the coefficients.

Another feature of using the Schur function expansions for Thom polynomials is that in all known to us cases, all the coefficients are nonnegative. In fact, we state the following "positivity conjecture":

Conjecture: The coefficients of the Schur function expansion of a Thom polynomial are nonnegative 1

To be more precise, we use here (the specializations of) supersymmetric Schur functions, also called "Schur functions in difference of alphabets" together with their three basic properties: vanishing, cancellation and factorization, (cf. [34, 3], [18, 24], 29], [19, 99, and [16]). These functions contain resultants among themselves. Their geometric role was illuminated, e.g., in the study of ideals of polynomials supported on degeneracy loci of [23], i.e. $\mathcal{P}$-ideals of singularities $\Sigma^{i}$ in the terminology of of the present paper (cf. the end of Section 2 and Theorem 11).

The main goal of this paper is to give the Thom polynomials for the singularities $I_{2,2}$ (in Mather's notation) associated with maps $\left(\mathbf{C}^{\bullet}, 0\right) \rightarrow$ $\left(\mathbf{C}^{\bullet+k}, 0\right)$ with parameter $k \geq 0$. We do it via establishing the Schur function expansions for these Thom polynomials. We prove first in Lemma 12 that the partitions appearing nontrivially have not more than 3 parts. Then, in Lemma 14, we establish a recursive relation for Thom polynomials associated with the successive values of the parameter $k$. This reduces our calculation to compute the (sub)sum indexed by partitions with precisely 2 parts. This is essentially done in Proposition 16 (see also Propositions 17, 18, 19).

Our main result (Theorem 20), combined with Propositions 18 and 19, gives an explicit presentation of the Thom polynomial for the singularities

\footnotetext{
${ }^{1}$ Note added in May 2006: this conjecture (formulated also by Feher and Komuves [6]) has been recently proved by Weber and the author in [30].
} 
$I_{2,2}$ with parameter $k \geq 0$ as a $\mathbf{Z}$-combination of Schur functions. We give closed algebraic expressions for the coefficients of these expansions. It turns out that these coefficients are the same as the coefficients of the Schur function expansions of the Segre classes of the second symmetric power of a rank 2 vector bundle, computed in [33], 23], [15], and [25].

Our main result offers a generalization (to any $k \geq 0$ ) of the formulas obtained previously by Porteous [21] and Rimanyi [31] for $k=0$ and $k=1$, respectively.

In our calculations, we use extensively the functorial $\lambda$-ring approach to symmetric functions developed mainly in Lascoux's book [16].

Main results of the present paper were announced in [26].

The forthcoming author's articles [27] and [28] will be devoted to study the Schur function expansions of Thom polynomials for the Morin's singularities $A_{i}$. Inspired by the present paper, [26], [27], and [28], Ozer Ozturk [20] computed the Thom polynomials for $A_{4}$ and $k=2,3$.

\section{Recollections on Thom polynomials}

Our main reference for this section is [31. We start with recalling what we shall mean by a "singularity". Let $k \geq 0$ be a fixed integer. By a singularity we shall mean an equivalence class of stable germs $\left(\mathbf{C}^{\bullet}, 0\right) \rightarrow\left(\mathbf{C}^{\bullet+k}, 0\right)$, where $\bullet \in \mathbf{N}$, under the equivalence generated by right-left equivalence (i.e. analytic reparametrizations of the source and target) and suspension (by suspension of a germ $\kappa$ we mean its trivial unfolding: $(x, v) \mapsto(\kappa(x), v))$.

We recall 2 that the Thom polynomial $\mathcal{T}^{\eta}$ of a singularity $\eta$ is a polynomial in the formal variables $c_{1}, c_{2}, \ldots$ that after the substitution

$$
c_{i}=c_{i}\left(f^{*} T Y-T X\right)=\left[c\left(f^{*} T Y\right) / c(T X)\right]_{i},
$$

for a general map $f: X \rightarrow Y$ between complex analytic manifolds, evaluates the Poincare dual of $\left[V^{\eta}(f)\right]$, where $V^{\eta}(f)$ is the cycle carried by the closure of the set

$$
\{x \in X \text { : the singularity of } f \text { at } x \text { is } \eta\} .
$$

By codimension of a singularity $\eta, \operatorname{codim}(\eta)$, we shall mean $\operatorname{codim}_{X}\left(V^{\eta}(f)\right)$ for such an $f$. The concept of the polynomial $\mathcal{T}^{\eta}$ comes from Thom's fundamental paper [35]. For a detailed discussion of the existence of Thom polynomials, see, e.g., [1. Thom polynomials associated with group actions were studied by Kazarian in [11, [12].

In fact, the above is the case with singularities without moduli [11, 7], [2]. The singularities $I_{2,2}$, studied in the present paper, have this property

\footnotetext{
${ }^{2}$ This statement is usually called the Thom-Damon theorem [35], 4].
} 
for $k \geq 0$. Indeed, the moduli of singularities start at codimension greater than $6 k+8$ (loc.cit.) whereas $\operatorname{codim}\left(I_{2,2}\right)=3 k+4$.

According to Mather's classification, singularities are in one-to-one correspondence with finite dimensional $\mathbf{C}$-algebras. We shall use the following notation:

- $A_{i}$ (of Thom-Boardman type $\Sigma^{1_{i}}$ ) will stand for the stable germs with local algebra $\mathbf{C}[[x]] /\left(x^{i+1}\right), i \geq 0$;

- $I_{2,2}$ (of Thom-Boardman type $\Sigma^{2}$ ) for stable germs with local algebra $\mathbf{C}[[x, y]] /\left(x y, x^{2}+y^{2}\right)$;

- III $I_{2,2}$ (of Thom-Boardman type $\Sigma^{2}$ ) for stable germs with local algebra $\mathbf{C}[[x, y]] /\left(x y, x^{2}, y^{2}\right)$, here $k \geq 1$.

In the present article, the computations of Thom polynomials shall use the method which stems from a sequence of papers by Rimanyi et al. [32, [31, 7], 2]. We sketch briefly this approach, refering the interested reader for more details to these papers, the main references being the last three mentioned items.

Let $k \geq 0$ be a fixed integer, and let $\eta:\left(\mathbf{C}^{\bullet}, 0\right) \rightarrow\left(\mathbf{C}^{\bullet+k}, 0\right)$ be a singularity with a prototype $\kappa:\left(\mathbf{C}^{n}, 0\right) \rightarrow\left(\mathbf{C}^{n+k}, 0\right)$. The maximal compact subgroup of the right-left symmetry group

$$
\text { Aut } \kappa=\left\{(\varphi, \psi) \in \operatorname{Diff}\left(\mathbf{C}^{n}, 0\right) \times \operatorname{Diff}\left(\mathbf{C}^{n+k}, 0\right): \psi \circ \kappa \circ \varphi^{-1}=\kappa\right\}
$$

of $\kappa$ will be denoted by $G_{\eta}$. Even if Aut $\kappa$ is much too large to be a finite dimensional Lie group, the concept of its maximal compact subgroup (up to conjugacy) can be defined in a sensible way (cf. [10] and [36]). In fact, $G_{\eta}$ can be chosen so that the images of its projections to the factors $\operatorname{Diff}\left(\mathbf{C}^{n}, 0\right)$ and $\operatorname{Diff}\left(\mathbf{C}^{n+k}, 0\right)$ are linear. Its representations via the projections on the source $\mathbf{C}^{n}$ and the target $\mathbf{C}^{n+k}$ will be denoted by $\lambda_{1}(\eta)$ and $\lambda_{2}(\eta)$. The vector bundles associated with the universal principal $G_{\eta}$-bundle $E G_{\eta} \rightarrow B G_{\eta}$ using the representations $\lambda_{1}(\eta)$ and $\lambda_{2}(\eta)$ will be called $E_{\eta}^{\prime}$ and $E_{\eta}$. The total Chern class of the singularity $\eta$ is defined in $H^{*}\left(B G_{\eta}, \mathbf{Z}\right)$ by

$$
c(\eta):=\frac{c\left(E_{\eta}\right)}{c\left(E_{\eta}^{\prime}\right)}
$$

The Euler class of $\eta$ is defined in $H^{2 \operatorname{codim}(\eta)}\left(B G_{\eta}, \mathbf{Z}\right)$ by

$$
e(\eta):=e\left(E_{\eta}^{\prime}\right) .
$$

In the following theorem, we collect information from [31], Theorem 2.4 and [7], Theorem 3.5, needed for the calculations in the present paper. 
Theorem 1 Suppose, for a singularity $\eta$, that the Euler classes of all singularities of smaller codimension than $\operatorname{codim}(\eta)$, are not zero-divisors 3 . Then we have

(i) if $\xi \neq \eta$ and $\operatorname{codim}(\xi) \leq \operatorname{codim}(\eta)$, then $\mathcal{T}^{\eta}(c(\xi))=0$;

(ii) $\mathcal{T}^{\eta}(c(\eta))=e(\eta)$.

This system of equations (taken for all such $\xi$ 's) determines the Thom polynomial $\mathcal{T}^{\eta}$ in a unique way.

To use this method of determining the Thom polynomials for singularities, one needs their classification, see, e.g., [5].

In Section 4, we shall use these equations to compute Thom polynomials. Sometimes, it is convenient not to work with the whole maximal compact subgroup $G_{\eta}$ but with its suitable subgroup; this subgroup should be, however, as "close" to $G_{\eta}$ as possible (cf. [31, p. 502). We shall denote this subgroup by the same symbol $G_{\eta}$. We recall the following recipe for computing maximal compact subgroups from [31 pp. 505-507. Let $\eta$ be a singularity whose prototype is $\kappa:\left(\mathbf{C}^{n}, 0\right) \rightarrow\left(\mathbf{C}^{n+k}, 0\right)$. The germ $\kappa$ is the miniversal unfolding of another germ $\beta:\left(\mathbf{C}^{m}, 0\right) \rightarrow\left(\mathbf{C}^{m+k}, 0\right)$ with $d \beta=0$. The group $G_{\eta}$ is a subgroup of the maximal compact subgroup of the algebraic automorphism group of the local algebra $Q_{\eta}$ of $\eta$ times the unitary group $U(k-d)$, where $d$ is the difference between the minimal number of relations and the number of generators of $Q_{\eta}$. With $\beta$ well chosen, $G_{\eta}$ acts as right-left symmetry group on $\beta$ with representations $\mu_{1}$ and $\mu_{2}$. The representations $\lambda_{1}$ and $\lambda_{2}$ are

$$
\lambda_{1}=\mu_{1} \oplus \mu_{V} \text { and } \lambda_{2}=\mu_{2} \oplus \mu_{V},
$$

where $\mu_{V}$ is the representation of $G_{\eta}$ on the unfolding space $V=\mathbf{C}^{n-m}$ given, for $\alpha \in V$ and $(\varphi, \psi) \in G_{\eta}$, by

$$
(\varphi, \psi) \alpha=\psi \circ \alpha \circ \varphi^{-1}
$$

For example, for the singularity of type $A_{i}:\left(\mathbf{C}^{\bullet}, 0\right) \rightarrow\left(\mathbf{C}^{\bullet+k}, 0\right)$, we have $G_{A_{i}}=U(1) \times U(k)$ with

$$
\mu_{1}=\rho_{1}, \quad \mu_{2}=\rho_{1}^{i+1} \oplus \rho_{k}, \quad \mu_{V}=\oplus_{j=2}^{i} \rho_{1}^{j} \oplus \oplus_{j=1}^{i}\left(\rho_{k} \otimes \rho_{1}^{-1}\right),
$$

where $\rho_{j}$ denotes the standard representation of the unitary group $U(j)$. Hence, we obtain assertion (i) of the following

Proposition 2 (i) Let $\eta=A_{i}$. For any $k$, writing $x$ and $y_{1}, \ldots, y_{k}$ for the Chern roots of the universal bundles on $B U(1)$ and $B U(k)$,

$$
c\left(A_{i}\right)=\frac{1+(i+1) x}{1+x} \prod_{j=1}^{k}\left(1+y_{j}\right) .
$$

\footnotetext{
${ }^{3}$ This is the so-called "Euler condition" (loc.cit.). The Euler condition holds true for the singularities $I_{2,2}$ for any $k \geq 0$.
} 
(ii) Let $\eta=I_{2,2}$. Denote by $H$ the extension of $U(1) \times U(1)$ by $\mathbf{Z} / 2 \mathbf{Z}$ ("the group generated by multiplication on the coordinates and their exchange"). For $k \geq 0, G_{\eta}=H \times U(k)$. Hence, for the purpose of our computations, we can use $G_{\eta}=U(1) \times U(1) \times U(k)$. Writing $x_{1}, x_{2}$ and $y_{1}, \ldots, y_{k}$ for the Chern roots of the universal bundles on two copies of $B U(1)$ and on $B U(k)$, we have

$$
\begin{gathered}
c\left(I_{2,2}\right)=\frac{\left(1+2 x_{1}\right)\left(1+2 x_{2}\right)}{\left(1+x_{1}\right)\left(1+x_{2}\right)} \prod_{j=1}^{k}\left(1+y_{j}\right) \\
e\left(I_{2,2}\right)=x_{1} x_{2}\left(x_{1}-2 x_{2}\right)\left(x_{2}-2 x_{1}\right) \prod_{j=1}^{k}\left(x_{1}-y_{j}\right)\left(x_{2}-y_{j}\right)\left(x_{1}+x_{2}-y_{j}\right) .
\end{gathered}
$$

(iii) Let $\eta=I I I_{2,2}$. For $k \geq 1, G_{\eta}=U(2) \times U(k-1)$, and writing $x_{1}, x_{2}$ and $y_{1}, \ldots, y_{k-1}$ for the Chern roots of the universal bundles on $B U(2)$ and $B U(k-1)$,

$$
c\left(I I I_{2,2}\right)=\frac{\left(1+2 x_{1}\right)\left(1+2 x_{2}\right)\left(1+x_{1}+x_{2}\right)}{\left(1+x_{1}\right)\left(1+x_{2}\right)} \prod_{j=1}^{k-1}\left(1+y_{j}\right) .
$$

(Assertions (ii) and (iii) are obtained, in a standard way, following the instructions of [31, Sect. 4. As for assertion (ii), compare [31, pp. 506-507] whereas assertion (iii) stems from [2, p. 65].)

Let $\eta$ be a singularity. As it was illuminated in the author's paper [23], in the case of the singularities $\eta=\Sigma^{i}$, it is natural and useful to consider a certain (homogeneous) ideal in the polynomial ring $R=\mathbf{Z}\left[c_{1}, c_{2}, \ldots\right]$ whose component of minimal degree is generated by $\mathcal{T}^{\eta}$. Namely, we denote by $\mathcal{P}^{\eta}$ the ideal of polynomials in $R$ which - after the substitution (1) - are supported on $V^{\eta}(f)$, where $f: X \rightarrow Y$ is a general map between complex analytic manifolds. (The notion of a "polynomial supported on a subscheme" can be found in [9], Appendix A.) Keeping track of [23, we shall call $\mathcal{P}^{\eta}$ the $\mathcal{P}$-ideal of the singularity $\eta$. For example, the $\mathcal{P}$-ideal of the singularity

$$
\Sigma^{i}:\left(\mathbf{C}^{m}, 0\right) \rightarrow\left(\mathbf{C}^{n}, 0\right)
$$

is

$$
\mathcal{P}^{\Sigma^{i}}=\mathcal{P}_{m-i},
$$

where on the RHS we have the ideal studied extensively in [23] (cf. also [22], [24]). We shall use this ideal in the proof of Theorem 11

In the present paper, it will be more handy to use, instead of $k$, a "shifted" parameter

$$
r:=k+1 \text {. }
$$

Sometimes, we shall write $\eta(r)$ for the singularity $\eta:\left(\mathbf{C}^{\bullet}, 0\right) \rightarrow\left(\mathbf{C}^{\bullet+r-1}, 0\right)$, and denote the Thom polynomial of $\eta(r)$ by $\mathcal{T}_{r}^{\eta}$ - to emphasize the dependence of both items on $r$. 
We have

$$
c_{i}\left(f^{*} T Y-T X\right)=S_{i}\left(T X^{*}-f^{*}\left(T Y^{*}\right)\right),
$$

where $S_{i}$ means the Segre class. We shall follow of the notation on the RHS and use, more generally, Schur functions $S_{\left(i_{1}, i_{2}, \ldots, i_{h}\right)}$, indexed by partitions, cf. the next section.

\section{Recollections on Schur functions}

In this section, we collect needed notions related to symmetric functions. We adopt the functorial point of view of [16] for what concerns symmetric functions. Namely, given a commutative ring, we treat symmetric functions as operators acting on the ring. (Here, these commutative rings are mostly Z-algebras generated by the Chern roots of the vector bundles from Proposition 2.)

Definition 3 By an alphabet $\mathbb{A}$, we understand a (finite) multi-set of elements in a commutative ring.

For $m \in \mathbf{N}$, by "an alphabet $\mathbb{A}_{m}$ " we shall mean an alphabet $\mathbb{A}=\left(a_{1}, \ldots, a_{m}\right)$ (of cardinality $m$ ); ditto for $\mathbb{B}_{n}=\left(b_{1}, \ldots, b_{n}\right), \mathbb{Y}_{k}=\left(y_{1}, \ldots, y_{k}\right)$, and $\mathbb{X}_{2}=\left(x_{1}, x_{2}\right)$.

Definition 4 Given two alphabets $\mathbb{A}, \mathbb{B}$, the complete functions $S_{i}(\mathbb{A}-\mathbb{B})$ are defined by the generating series (with $z$ an extra variable):

$$
\sum S_{i}(\mathbb{A}-\mathbb{B}) z^{i}=\prod_{b \in \mathbb{B}}(1-b z) / \prod_{a \in \mathbb{A}}(1-a z) .
$$

So $S_{i}(\mathbb{A}-\mathbb{B})$ interpolates between $S_{i}(\mathbb{A})$ - the complete homogeneous symmetric function of degree $i$ in $\mathbb{A}$ and $S_{i}(-\mathbb{B})$ - the $i$ th elementary function in $\mathbb{B}$ times $(-1)^{i}$. The notation $\mathbb{A}-\mathbb{B}$ is compatible with the multiplication of series

$$
\sum S_{i}(\mathbb{A}-\mathbb{B}) z^{i} \cdot \sum S_{j}\left(\mathbb{A}^{\prime}-\mathbb{B}^{\prime}\right) z^{j}=\sum S_{i}\left(\left(\mathbb{A}+\mathbb{A}^{\prime}\right)-\left(\mathbb{B}+\mathbb{B}^{\prime}\right)\right) z^{i},
$$

the sum $\mathbb{A}+\mathbb{A}^{\prime}$ denoting the union of two alphabets $\mathbb{A}$ and $\mathbb{A}^{\prime}$.

Convention 5 We shall often identify an alphabet $\mathbb{A}=\left\{a_{1}, \ldots, a_{m}\right\}$ with the sum $a_{1}+\cdots+a_{m}$ and perform usual algebraic operations on such elements. For example, $\mathbb{A} b$ will denote the alphabet $\left(a_{1} b, \ldots, a_{m} b\right)$. We will give priority to the algebraic notation over the set-theoretic one. In fact, in the following, we shall use mostly alphabets of variables.

We have $(\mathbb{A}+\mathbb{C})-(\mathbb{B}+\mathbb{C})=\mathbb{A}-\mathbb{B}$, and this corresponds to simplification of the common factor for the rational series:

$$
\sum S_{i}((\mathbb{A}+\mathbb{C})-(\mathbb{B}+\mathbb{C})) z^{i}=\sum S_{i}(\mathbb{A}-\mathbb{B}) z^{i} .
$$


Definition 6 By a partition $I=\left(i_{1}, i_{2}, \ldots, i_{h}\right)$ we mean a weakly increasing sequence $0 \leq i_{1} \leq i_{2} \leq \ldots \leq i_{h}$ of natural numbers.

In the following, we shall identify partitions with their Young diagrams, as is customary.

Definition 7 Given a partition $I$ and two alphabets $\mathbb{A}$ and $\mathbb{B}$, the Schur function $S_{I}(\mathbb{A}-\mathbb{B})$ is defined by the following determinant:

$$
S_{I}(\mathbb{A}-\mathbb{B}):=\left|S_{i_{p}+p-q}(\mathbb{A}-\mathbb{B})\right|_{1 \leq p, q \leq h} .
$$

These functions are often called supersymmetric Schur functions or Schur functions in difference of alphabets. Their properties were studied, among others, in [34], 3], 18], 24], 29], [19], [9], and [16]. From the last item, we borrow a use of increasing "French" partitions and the determinant of the form (17) evaluating a Schur function. We shall use the simplified notation $i_{1} i_{2} \cdots i_{h}$ or $i_{1}, i_{2}, \ldots, i_{h}$ for a partition $\left(i_{1}, i_{2}, \ldots, i_{h}\right)$ (the latter one if $i_{h} \geq$ 10). The rectangle partition $(i, i, \ldots, i)$ ( $h$ times) will be denoted $\left(i^{h}\right)$.

For example,

$$
S_{33344}(\mathbb{A}-\mathbb{B})=\left|\begin{array}{ccccc}
S_{3} & S_{4} & S_{5} & S_{7} & S_{8} \\
S_{2} & S_{3} & S_{4} & S_{6} & S_{7} \\
S_{1} & S_{2} & S_{3} & S_{5} & S_{6} \\
1 & S_{1} & S_{2} & S_{4} & S_{5} \\
0 & 1 & S_{1} & S_{3} & S_{4}
\end{array}\right|
$$

where $S_{i}$ means $S_{i}(\mathbb{A}-\mathbb{B})$.

We shall now give some properties of Schur functions. The details can be found in the just quoted references. By Eq. (16), we get the following cancellation property:

$$
S_{I}((\mathbb{A}+\mathbb{C})-(\mathbb{B}+\mathbb{C}))=S_{I}(\mathbb{A}-\mathbb{B}) .
$$

We record the following property justifying the notational remark from the end of Section 2; for a partition $I$,

$$
S_{I}(\mathbb{A}-\mathbb{B})=(-1)^{|I|} S_{J}(\mathbb{B}-\mathbb{A})=S_{J}\left(\mathbb{B}^{*}-\mathbb{A}^{*}\right),
$$

where $J$ is the conjugate partition of $I$ (i.e. the consecutive rows of $J$ are equal to the corresponding columns of $I)$, and $\mathbb{A}^{*}$ denotes the alphabet $\left\{-a_{1},-a_{2}, \ldots\right\}$.

Fix two positive integers $m$ and $n$. We shall say that a partition $I=$ $\left(i_{1}, i_{2}, \ldots, i_{h}\right)$ is contained in the $(m, n)$-hook if either $h \leq m$, or $h>m$ and $i_{h-m} \leq n$. Pictorially, this means that the Young diagram of $I$ is contained in the "tickened" hook 


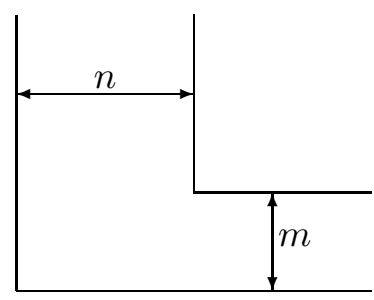

We record the following vanishing property. Given alphabets $\mathbb{A}$ and $\mathbb{B}$ of cardinalities $m$ and $n$, if a partition $I$ is not contained in the $(m, n)$-hook, then

$$
S_{I}(\mathbb{A}-\mathbb{B})=0 .
$$

For example,

$$
S_{3569}\left(\mathbb{A}_{2}-\mathbb{B}_{4}\right)=S_{3569}\left(a_{1}+a_{2}-b_{1}-b_{2}-b_{3}-b_{4}\right)=0
$$

because 3569 is not contained in the $(2,4)$-hook. In fact, we have the following result.

Theorem 8 If $\mathbb{A}_{m}$ and $\mathbb{B}_{n}$ are alphabets of variables, then the functions $S_{I}\left(\mathbb{A}_{m}-\mathbb{B}_{n}\right)$, for I running over partitions contained in the $(m, n)$-hook, are $\mathbf{Z}$-linearly independent.

(They form a $\mathbf{Z}$-basis of the abelian group of the so-called "supersymmetric functions".)

In the present paper, by a symmetric function we shall mean a $\mathbf{Z}$-linear combination of the operators $S_{I}(-)$.

Definition 9 Given two alphabets $\mathbb{A}, \mathbb{B}$, we define their resultant

$$
R(\mathbb{A}, \mathbb{B}):=\prod_{a \in \mathbb{A}, b \in \mathbb{B}}(a-b) .
$$

This terminology is justified by the fact that $R(\mathbb{A}, \mathbb{B})$ is the classical resultant of the polynomials $R(x, \mathbb{A})$ and $R(x, \mathbb{B})$. We have

$$
R\left(\mathbb{A}_{m}, \mathbb{B}_{n}\right)=S_{\left(n^{m}\right)}(\mathbb{A}-\mathbb{B})=\sum_{I} S_{I}(\mathbb{A}) S_{\left(n^{m}\right) / I}(-\mathbb{B}),
$$

where the sum is over all partitions $I \subset\left(n^{m}\right)$.

When a partition is contained in the $(m, n)$-hook and at the same time it contains the rectangle $\left(n^{m}\right)$, then we have the following factorization property: for partitions $I=\left(i_{1}, \ldots, i_{m}\right)$ and $J=\left(j_{1}, \ldots, j_{h}\right)$,

$$
S_{\left(j_{1}, \ldots, j_{h}, i_{1}+n, \ldots, i_{m}+n\right)}\left(\mathbb{A}_{m}-\mathbb{B}_{n}\right)=S_{I}(\mathbb{A}) R(\mathbb{A}, \mathbb{B}) S_{J}(-\mathbb{B}) .
$$

The following convention stems from Lascoux's paper [17]. 
Convention 10 We may need to specialize a letter to 2 , but this must not be confused with taking two copies of 1 . To allow one, nevertheless, specializing a letter to an (integer, or even complex) number $r$ inside a symmetric function, without introducing intermediate variables, we write $r$ for this specialization. Boxes have to be treated as single variables. For example, $S_{i}(2)=\left(\begin{array}{c}i+1 \\ 2\end{array}\right)$ but $S_{i}(2)=2^{i}$. A similar remark applies to $\mathbf{Z}$-linear combinations of variables. We have $S_{2}\left(\mathbb{X}_{2}\right)=x_{1}^{2}+x_{1} x_{2}+x_{2}^{2}$ but $S_{2}\left(x_{1}+x_{2}\right)=x_{1}^{2}+2 x_{1} x_{2}+x_{2}^{2}, S_{11}\left(\mathbb{X}_{2}\right)=x_{1} x_{2}$ but $S_{11}\left(x_{1}+x_{2}\right)=0$, $S_{2}(3 x)=6 x^{2}$ but $S_{2}(3 x)=9 x^{2}$ etc.

For example,

$$
\prod_{j=1}^{k}\left(x_{1}-y_{j}\right)\left(x_{2}-y_{j}\right)\left(x_{1}+x_{2}-y_{j}\right)=R\left(\mathbb{X}_{2}+x_{1}+x_{2}, \mathbb{Y}_{k}\right)
$$

This convention will be used in the next section.

We end the present section with the following result which is a consequence of the author's study [23], 22], 24] of the $\mathcal{P}$-ideals of the singularities $\Sigma^{i}$.

Theorem 11 Suppose that a singularity $\eta$ is of Thom-Boardman type $\Sigma^{i}$. Then all summands in the Schur function expansion of $\mathcal{T}_{r}^{\eta}$ are indexed by partitions containing the rectangle partition $(r+i-1)^{i}$.

Proof. Since $\eta$ is of Thom-Boardman type $\Sigma^{i}$, the Thom polynomial $\mathcal{T}_{r}^{\eta}$ belongs to the $\mathcal{P}$-ideal of the singularity $\Sigma^{i}$ with parameter $r$. We also know by the Thom-Damon theorem (cf. [4]) that $\mathcal{T}_{r}^{\eta}$ is a $\mathbf{Z}$-linear combination of Schur functions in $T X^{*}-f^{*}\left(T Y^{*}\right)$. The assertion now follows by combining Theorem 3.4 from [23] with Lemma 2.5 from [22] (see also Claim in the proof of Theorem 5.3(i) in [24]). Indeed, it follows from the former result that any $\mathbf{Z}$-combination of Schur functions indexed by partitions containing $(r+i-1)^{i}$ belongs to $\mathcal{P}^{\Sigma_{i}(r)}$, whereas the latter result implies that no nonzero Z-combination $\sum_{I} \alpha_{I} S_{I}$, where all $I \not \supset(r+i-1)^{i}$, belongs to $\mathcal{P}^{\Sigma_{i}(r)}$.

\section{Thom polynomial for $I_{2,2}(r)$}

The codimension of $I_{2,2}(r), r \geq 1$, is $3 r+1$. The Thom polynomial for $I_{2,2}(1)$ is $S_{22}=S_{22}\left(T X^{*}-f^{*}\left(T Y^{*}\right)\right.$ ) (cf. [21]). In the following, we shall often omit the arguments of Schur functions.

\footnotetext{
${ }^{4}$ We say that one partition is contained in another if this holds for their Young diagrams (cf. [16]).
} 
From now on, we shall assume that $r \geq 2$. The Thom polynomial for $I_{2,2}(2)$ is (cf. [31])

$$
S_{133}+3 S_{34}
$$

By virtue of Proposition 2, the equations from Theorem 1 characterizing the Thom polynomial for $I_{2,2}(r)$ are

$$
P\left(-\mathbb{B}_{r-1}\right)=P\left(x-2 x-\mathbb{B}_{r-1}\right)=P\left(x-3 x-\mathbb{B}_{r-1}\right)=0,
$$

and (using Eq. (24) )

$$
P\left(\mathbb{X}_{2}-2 x_{1}-2 x_{2}-\mathbb{B}_{r-1}\right)=x_{1} x_{2}\left(x_{1}-2 x_{2}\right)\left(x_{2}-2 x_{1}\right) R\left(\mathbb{X}_{2}+x_{1}+x_{2}, \mathbb{B}_{r-1}\right) .
$$

Here, without loss of generality, we assume that $x, x_{1}, x_{2}$, and $\mathbb{B}_{r-1}$ are variables. Moreover, $P(-)$ denotes a symmetric function. For the remainder of this paper, we set

$$
\mathbb{D}:=2 x_{1}+2 x_{2}+x_{1}+x_{2} .
$$

Then, additionally, for variables $x_{1}, x_{2}$ and an alphabet $\mathbb{B}_{r-2}$, we have the vanishing imposed by $I I I_{2,2}$ :

$$
P\left(\mathbb{X}_{2}-\mathbb{D}-\mathbb{B}_{r-2}\right)=0 .
$$

Indeed, the singularities $\neq I_{2,2}$ with codimension $\leq \operatorname{codim}\left(I_{2,2}\right)$ are: $A_{0}, A_{1}$, $A_{2}, I I I_{2,2}$.

For $r \geq 1$, we set

$$
\mathcal{T}_{r}:=\mathcal{T}_{r}^{I_{2,2}} .
$$

Our goal is to give a presentation of $\mathcal{T}_{r}$ as a $\mathbf{Z}$-linear combination of Schur functions with explicit algebraic expressions of the coefficients:

$$
\mathcal{T}_{r}=\sum_{I} \alpha_{I} S_{I}
$$

We shall say that a partition $I$ appears nontrivially in Eq. (30) if $\alpha_{I} \neq 0$.

Lemma 12 (i) A partition appearing nontrivially in the Schur function expansion of $\mathcal{T}_{r}$ contains the partition $(r+1, r+1)$.

(ii) A partition appearing nontrivially in the Schur function expansion of $\mathcal{T}_{r}$ has at most three parts.

Proof. (i) Since the singularity $I_{2,2}$ is of Thom-Boardman type $\Sigma^{2}$, this is a particular case of Theorem [11.

(ii) We can assume that $r \geq 3$. In addition to information contained in (i), we shall use Eq. (28)

$$
\mathcal{T}_{r}\left(\mathbb{X}_{2}-\mathbb{D}-\mathbb{B}_{r-2}\right)=0
$$


By virtue of (i), we can use factorization property (23) to all summands of

$$
\mathcal{T}_{r}\left(\mathbb{X}_{2}-\mathbb{D}-\mathbb{B}_{r-2}\right)=\sum_{I} \alpha_{I} S_{I}\left(\mathbb{X}_{2}-\mathbb{D}-\mathbb{B}_{r-2}\right)
$$

(we assume that $\alpha_{I} \neq 0$ ). We divide each summand of this last polynomial by the resultant

$$
R\left(\mathbb{X}_{2}, \mathbb{D}+\mathbb{B}_{r-2}\right) .
$$

Suppose that the resulting factor of $S_{I}$ is

$$
S_{p, q}\left(\mathbb{X}_{2}\right) S_{J}\left(-\mathbb{D}-\mathbb{B}_{r-2}\right),
$$

cf. (23). Since $|I|=3 r+1$, we have

$$
|J| \leq r-1 .
$$

Now, let us assume that $I$ has more than 3 parts, that is, $J$ has 2 or more parts. This assumption (together with the inequality (33)) implies that

$$
S_{J}\left(-\mathbb{B}_{r-2}\right) \neq 0
$$

$\left(\mathbb{B}_{r-2}\right.$ is an alphabet of variables). Expanding (32), we get among summands the following one of largest possible degree $|J|$ in $\mathbb{B}_{r-2}$ :

$$
S_{p, q}\left(\mathbb{X}_{2}\right) S_{J}\left(-\mathbb{B}_{r-2}\right) \neq 0 .
$$

Take in the sum

$$
\sum_{I} \alpha_{I} S_{p, q}\left(\mathbb{X}_{2}\right) S_{J}\left(-\mathbb{D}-\mathbb{B}_{r-2}\right)
$$

the (sub)sum of all the nonzero summands of the form (32) with the largest possible weight of $J$. Since Schur polynomials are independent, this (sub)sum is nonzero, and moreover, it is $\mathbf{Z}$-linearly independent of other summands both in the sum indexed by partitions $I$ with $\geq 3$ parts, and in that indexed by partitions with 2 parts (this last sum does not depend on $\mathbb{B}_{r-2}$ ). Hence, there is no Z-linear combination of $S_{I}$ 's involving nontrivially $I$ with more than 3 parts (and possibly also those with 3 or 2 parts) that satisfies Eq. (28). Assertion (ii) has been proved.

(For example, $S_{1144}$ cannot appear in the Schur function expansion of $\mathcal{T}_{3}$ because $S_{1144}\left(\mathbb{X}_{2}-\mathbb{D}-\mathbb{B}_{1}\right)$ after division by the resultant contains the summand $S_{11}\left(-\mathbb{B}_{1}\right)=S_{2}\left(\mathbb{B}_{1}\right)$, which does not occur in similar expressions for $S_{55}, S_{46}, S_{244}, S_{145}$.)

Corollary 13 If $S_{i_{1}, i_{2}}$ appears nontrivially in the Schur function expansion of $\mathcal{T}_{r}$, then $i_{1}=r+1+p$ and $i_{2}=2 r-p$, where $0 \leq 2 p \leq r-1$. 
The following lemma gives a recursive description of $\mathcal{T}_{r}$. Denote by $\Phi$ the linear endomorphism on the $\mathbf{Z}$-module spanned by Schur functions indexed by partitions of length $\leq 3$, that sends a Schur function $S_{i_{1}, i_{2}, i_{3}}$ to $S_{i_{1}+1, i_{2}+1, i_{3}+1}$. Let $\overline{\mathcal{T}_{r}}$ denote the sum of those terms in the Schur function expansion of $\mathcal{T}_{r}$ which are indexed by partitions of length $\leq 2$. Note that $\overline{\mathcal{T}}_{1}=\mathcal{T}_{1}=S_{22}$.

Lemma 14 With this notation, for $r \geq 2$, we have the following recursive equation:

$$
\mathcal{T}_{r}=\overline{\mathcal{T}}_{r}+\Phi\left(\mathcal{T}_{r-1}\right)
$$

Proof. Write

$$
\mathcal{T}_{r}=\sum_{I} \alpha_{I} S_{I}=\sum_{J} \alpha_{J} S_{J}+\sum_{K} \alpha_{K} S_{K}
$$

where $J$ have 2 parts and $K=\left(k_{1}, k_{2}, k_{3}\right)$ have 3 parts (we assume that $\left.\alpha_{I} \neq 0\right)$. We set

$$
Q=\sum_{K} \alpha_{K} S_{k_{1}-1, k_{2}-1, k_{3}-1},
$$

and our goal is to show that $Q=\mathcal{T}_{r-1}$. Since a partition $I$ appearing nontrivially in the Schur function expansion of $\mathcal{T}_{r}$ must contain the partition $(r+1, r+1)$, any partition $\left(k_{1}-1, k_{2}-1, k_{3}-1\right)$ above contains the partition $(r, r)$. Since this last partition is not contained in the $(1, r-1)$-hook, Eqs. (25) with $r$ replaced by $r-1$ and $P$ by $Q$ are automatically fulfilled by virtue of the vanishing property (20). Note that Eq. (28) is a particular case of Eq. (26). Indeed, specializing $b_{r-1}$ to $x_{1}+x_{2}$ in Eq. (26), we get Eq. (28). Therefore it suffices to show that

$$
Q\left(\mathbb{X}_{2}-\mathbb{E}-\mathbb{B}_{r-2}\right)=x_{1} x_{2}\left(x_{1}-2 x_{2}\right)\left(x_{2}-2 x_{1}\right) R\left(\mathbb{X}_{2}+x_{1}+x_{2}, \mathbb{B}_{r-2}\right),
$$

where $\mathbb{E}=2 x_{1}+2 x_{2}$. We apply to each summand

$$
\alpha_{K} S_{k_{1}-1, k_{2}-1, k_{3}-1}\left(\mathbb{X}_{2}-\mathbb{E}-\mathbb{B}_{r-2}\right)
$$

of $Q\left(\mathbb{X}_{2}-\mathbb{E}-\mathbb{B}_{r-2}\right)$ the factorization property (23), and divide it by the resultant

$$
R\left(\mathbb{X}_{2}, \mathbb{E}+\mathbb{B}_{r-2}\right) .
$$

Suppose that the resulting factor is

$$
\alpha_{K} S_{a, b}\left(\mathbb{X}_{2}\right) S_{c}\left(-\mathbb{E}-\mathbb{B}_{r-2}\right),
$$

where $\left(k_{1}-1, k_{2}-1, k_{3}-1\right)=(c, r+a, r+b)$.

Performing the same division of

$$
x_{1} x_{2}\left(x_{1}-2 x_{2}\right)\left(x_{2}-2 x_{1}\right) R\left(\mathbb{X}_{2}+x_{1}+x_{2}, \mathbb{B}_{r-2}\right),
$$


we get $R\left(x_{1}+x_{2}, \mathbb{B}_{r-2}\right)$. Thus, the desired equation $Q=\mathcal{T}_{r-1}$ is equivalent to

$$
\sum_{a+b+c=r-2} \alpha_{K} S_{a, b}\left(\mathbb{X}_{2}\right) S_{c}\left(-\mathbb{E}-\mathbb{B}_{r-2}\right)=R\left(x_{1}+x_{2}, \mathbb{B}_{r-2}\right) .
$$

To prove Eq. (40), we use Eqs. (26) and (36) for $\mathcal{T}_{r}$

$$
\sum_{I} \alpha_{I} S_{I}\left(\mathbb{X}_{2}-\mathbb{E}-\mathbb{B}_{r-1}\right)=x_{1} x_{2}\left(x_{1}-2 x_{2}\right)\left(x_{2}-2 x_{1}\right) R\left(\mathbb{X}_{2}+x_{1}+x_{2}, \mathbb{B}_{r-1}\right) .
$$

Using again the factorization property (this time w.r.t. the larger rectangle $(r+1, r+1))$ and dividing both sides of the last equation by the resultant

$$
R\left(\mathbb{X}_{2}, \mathbb{E}+\mathbb{B}_{r-1}\right),
$$

we get the identity

$$
\sum_{p+q+j=r-1} \alpha_{I} S_{p, q}\left(\mathbb{X}_{2}\right) S_{j}\left(-\mathbb{E}-\mathbb{B}_{r-1}\right)=R\left(x x_{1}+x_{2}, \mathbb{B}_{r-1}\right) .
$$

Since

$$
S_{j}\left(-\mathbb{E}-\mathbb{B}_{r-1}\right)=S_{j}\left(-\mathbb{E}-\mathbb{B}_{r-2}\right)-b_{r-1} S_{j-1}\left(-\mathbb{E}-\mathbb{B}_{r-2}\right)
$$

and

$$
R\left(x_{1}+x_{2}, \mathbb{B}_{r-1}\right)=\left(x_{1}+x_{2}-b_{r-1}\right) R\left(x_{1}+x_{2}, \mathbb{B}_{r-2}\right),
$$

taking the coefficients of $\left(-b_{r-1}\right)$ in both sides of Eq. (41), we get the desired Eq. (40). The lemma has been proved.

(For example, writing $\mathcal{T}_{3}=\alpha S_{46}+\beta S_{55}+\gamma S_{244}+\delta S_{145}$, we get that

$$
\gamma S_{1}\left(-\mathbb{E}-B_{1}\right)+\delta S_{1}\left(\mathbb{X}_{2}\right)=R\left(x_{1}+x_{2}, \mathbb{B}_{1}\right),
$$

by taking the coeficients of $\left(-b_{2}\right)$ in both sides of

$\left.\alpha S_{2}\left(\mathbb{X}_{2}\right)+\beta S_{11}\left(\mathbb{X}_{2}\right)+\gamma S_{2}\left(-\mathbb{E}-\mathbb{B}_{2}\right)+\delta S_{1}\left(-\mathbb{E}-\mathbb{B}_{2}\right) S_{1}\left(X_{2}\right)=R\left(x_{1}+x_{2}, \mathbb{B}_{2}\right).\right)$

Iterating Eq. (35) gives

Corollary 15 With the above notation, we have

$$
\mathcal{T}_{r}=\overline{\mathcal{T}}_{r}+\Phi\left(\overline{\mathcal{T}}_{r-1}\right)+\Phi^{2}\left(\overline{\mathcal{T}}_{r-2}\right)+\cdots+\Phi^{r-1}\left(\overline{\mathcal{T}}_{1}\right) .
$$

Of course, $\overline{\mathcal{T}}_{r}$ is uniquely determined by its value on $\mathbb{X}_{2}$. The following result gives this value.

Proposition 16 For any $r \geq 1$, we have

$$
\overline{\mathcal{T}}_{r}\left(\mathbb{X}_{2}\right)=\left(x_{1} x_{2}\right)^{r+1} S_{r-1}(\mathbb{D}) .
$$


Proof. We use induction on $r$. For $r=1,2$, the assertion holds true. Suppose that the assertion is true for $\overline{\mathcal{T}}_{i}$ where $i<r$. Fix a partition $I=(j, r+1+p, r+1+q)$ appearing nontrivially in the Schur function expansion (30) of $\mathcal{T}_{r}$. Note that $j$ varies from 0 to $r-1$ because $|I|=3 r+1$. We obtain, by the factorization property (23),

$$
S_{I}\left(\mathbb{X}_{2}-\mathbb{D}-\mathbb{B}_{r-2}\right)=R \cdot S_{j}\left(-\mathbb{D}-\mathbb{B}_{r-2}\right) \cdot S_{p, q}\left(\mathbb{X}_{2}\right),
$$

where $R=R\left(\mathbb{X}_{2}, \mathbb{D}+\mathbb{B}_{r-2}\right)$. Hence, using Eq. (42), we see that

$$
\mathcal{T}_{r}\left(\mathbb{X}_{2}-\mathbb{D}-\mathbb{B}_{r-2}\right)=R \cdot\left(\sum_{j=0}^{r-1} S_{j}\left(-\mathbb{D}-\mathbb{B}_{r-2}\right) \frac{\overline{\mathcal{T}}_{r-j}\left(\mathbb{X}_{2}\right)}{\left(x_{1} x_{2}\right)^{r-j+1}}\right) .
$$

By the induction assumption, for positive $j \leq r-1$,

$$
\overline{\mathcal{T}}_{r-j}\left(\mathbb{X}_{2}\right)=\left(x_{1} x_{2}\right)^{r-j+1} S_{r-1-j}(\mathbb{D}) .
$$

Substituting this to (44), and using the vanishing (28), we obtain

$$
\sum_{j=1}^{r-1} S_{j}\left(-\mathbb{D}-\mathbb{B}_{r-2}\right) S_{r-1-j}(\mathbb{D})+\frac{\overline{\mathcal{T}}_{r}\left(\mathbb{X}_{2}\right)}{\left(x_{1} x_{2}\right)^{r+1}}=0 .
$$

But we also have, by a formula for addition of alphabets,

$$
\sum_{j=1}^{r-1} S_{j}\left(-\mathbb{D}-\mathbb{B}_{r-2}\right) S_{r-1-j}(\mathbb{D})+S_{r-1}(\mathbb{D})=S_{r-1}\left(-\mathbb{B}_{r-2}\right)=0 .
$$

Combining Eqs. (45) and (46) gives

$$
\overline{\mathcal{T}}_{r}\left(\mathbb{X}_{2}\right)=\left(x_{1} x_{2}\right)^{r+1} S_{r-1}(\mathbb{D}),
$$

that is, the induction assertion. The proof of the proposition is now complete.

The Schur function expansion of $S_{i}(\mathbb{D})$ was described in [23], [15], and [25. App. A3] in the context of the Segre classes of the second symmetric power of a rank 2 vector bundle. Indeed, $\mathbb{D}$ is the alphabet of the Chern roots of the second symmetric power of a rank 2 bundle with the Chern roots $x_{1}, x_{2}$.

Denote by $\langle p, q\rangle$ the coefficient of $S_{p, q}:=S_{p, q}\left(\mathbb{X}_{2}\right)$ in $S_{p+q}(\mathbb{D})$, where $0 \leq p \leq q$. A proof of the next proposition, due to Lascoux with the help of divided differences, can be found in [25], pp. 163-166. We give here another proof without divided differences.

Proposition 17 For $p>0$, we have

$$
\langle p, q\rangle=\langle p-1, q\rangle+\langle p, q-1\rangle .
$$


Proof. We have

$$
S_{i}(\mathbb{D})=\sum_{h=0}^{i} S_{h}\left(2 x_{1}+2 x_{2}\right) S_{i-h}\left(x_{1}+x_{2}\right)=\sum_{h=0}^{i} 2^{h} S_{h} \cdot\left(x_{1}+x_{2}\right)^{i-h},
$$

and (cf., e.g., [19] I.4, Ex.3)

$$
\left(x_{1}+x_{2}\right)^{j}=\sum_{a, b \geq 0}\left(\begin{array}{c}
a+b \\
a
\end{array}\right) \frac{b-a+1}{b+1} S_{a, b},
$$

where $a+b=j$ and $a \leq b$. Combining Eqs. (48), (49) with the Pieri formula (cf., e.g., [16], [19]), we get for $0 \leq p \leq q$,

$$
\langle p, q\rangle=\sum_{h=0}^{p+q} 2^{h} \sum_{h_{1}, h_{2} \geq 0}\left(\begin{array}{c}
p+q-h \\
p-h_{1}
\end{array}\right) \frac{\left(q-h_{2}\right)-\left(p-h_{1}\right)+1}{q-h_{2}+1},
$$

where $h_{1}+h_{2}=h$ and $h_{1} \leq p \leq q-h_{2}$.

We also compute the Schur function expansion of $S_{1, i-1}(\mathbb{D})$. Denote by $[p, q]$ the coefficient of $S_{p, q}$ in $S_{1, p+q-1}(\mathbb{D}), 0 \leq p \leq q$. We have the following expansion for $S_{1, i-1}(\mathbb{D})$ :

$$
\begin{aligned}
& \sum_{h=1}^{i} S_{(1, i-1) /(i-h)}\left(2 x_{1}+2 x_{2}\right) S_{h}\left(x_{1}+x_{2}\right) \\
& =\sum_{h=1}^{i} 2^{h} S_{h} \cdot\left(x_{1}+x_{2}\right)^{i-h}+\sum_{h=1}^{i} 2^{h} S_{1, h-1} \cdot\left(x_{1}+x_{2}\right)^{i-h} .
\end{aligned}
$$

We get from both sums in the last line that for $p>0$ the coefficient $[p, q]$ is equal twice the RHS of Eq. (50), that is,

$$
[p, q]=2\langle p, q\rangle .
$$

We have, by the Pieri formula,

$$
S_{i-1}(\mathbb{D}) \cdot S_{1}(\mathbb{D})=S_{i-1}(\mathbb{D}) \cdot 3 S_{1}=S_{i}(\mathbb{D})+S_{1, i-1}(\mathbb{D}) .
$$

This equation implies that $S_{p, q}$ appears in $S_{i}(\mathbb{D})+S_{1, i-1}(\mathbb{D})$ with multiplicity $3(\langle p-1, q\rangle+\langle p-1, q\rangle)$ (we use the Pieri formula once again). The desired Eq. (47) now follows by virtue of Eq. (51).

We now pass to some "closed" algebraic expressions for the $\langle p, q\rangle$ 's. We have

$$
\langle 0, q\rangle=S_{q}(1+2)=1+2+\cdots+2^{q}=2^{q+1}-1 .
$$

The following result was obtained in [33], 23], and [15]. 
Proposition 18 For $0 \leq p \leq q$, we have

$$
\langle p, q\rangle=\left(\begin{array}{c}
p+q+1 \\
p+1
\end{array}\right)+\left(\begin{array}{c}
p+q+1 \\
p+2
\end{array}\right)+\cdots+\left(\begin{array}{c}
p+q+1 \\
q+1
\end{array}\right) .
$$

We propose now an alternative expression involving powers of 2 , which is a natural generalization of the equation $\langle 0, q\rangle=2^{q+1}-1$, and which stems directly from Eq. (47). Namely, with the convention that $\left(\begin{array}{l}a \\ 0\end{array}\right)=1$ for any $a \in \mathbf{Z}$, we have

Proposition 19 For $0 \leq p \leq q$,

$$
\langle p, q\rangle=2^{p+q+1}-\sum_{s=0}^{p}\left[\left(\begin{array}{c}
p+q-2 s-1 \\
p-s
\end{array}\right)-\left(\begin{array}{c}
2 p-2 s-1 \\
p-s+1
\end{array}\right)\right] 2^{2 s} .
$$

Proof. The proof uses double induction on $p$ and $q$. We use Eq. (47) several times:

$$
\begin{aligned}
\langle p, q\rangle & =\langle p-1, q\rangle+\langle p, q-1\rangle \\
& =\langle p-1, q\rangle+\langle p-1, q-1\rangle+\langle p, q-2\rangle \\
& =\ldots \\
& =\langle p-1, q\rangle+\cdots+\langle p-1,1\rangle+\langle p, 0\rangle .
\end{aligned}
$$

We know the values of all summands in the last row by the induction assumption (the last summand being equal to $2^{p+1}-1$ ). Using several times Eq. (53) as well as a well-known equality:

$$
1+\left(\begin{array}{c}
a+1 \\
a
\end{array}\right)+\left(\begin{array}{c}
a+2 \\
a
\end{array}\right)+\cdots+\left(\begin{array}{c}
2 a-2 \\
a
\end{array}\right)=\left(\begin{array}{c}
2 a-1 \\
a+1
\end{array}\right),
$$

we get the desired induction assertion (55) for $\langle p, q\rangle$.

Using Proposition 16, we shall now give the Schur function expansion of $\overline{\mathcal{T}}_{r}$. Denote by $d_{r j}$ the coefficient of $S_{r+j, 2 r+1-j}$ in $\overline{\mathcal{T}}_{r}$ for $r \geq 1$ and $j \geq 1$. By virtue of Corollary [13, $d_{r j} \neq 0$ entails $j \leq[(r+1) / 2]$ (for example, the only Schur functions that can appear with nonzero coefficients in $\overline{\mathcal{T}}_{5}$ are $S_{6,10}, S_{79}$, and $\left.S_{88}\right)$, so that we have

$$
\overline{\mathcal{T}}_{r}=\sum_{j=1}^{[(r+1) / 2]} d_{r j} S_{r+j, 2 r+1-j} .
$$

We have the following link between the $d_{r j}$ 's and $\langle p, q\rangle$ 's: suppose that $d_{r j} \neq 0$, then we have

$$
d_{r j}=\langle j-1, r-j\rangle .
$$

We may display the $d_{r j}$ 's with the help of the following "Pascal triangle"type matrix: 


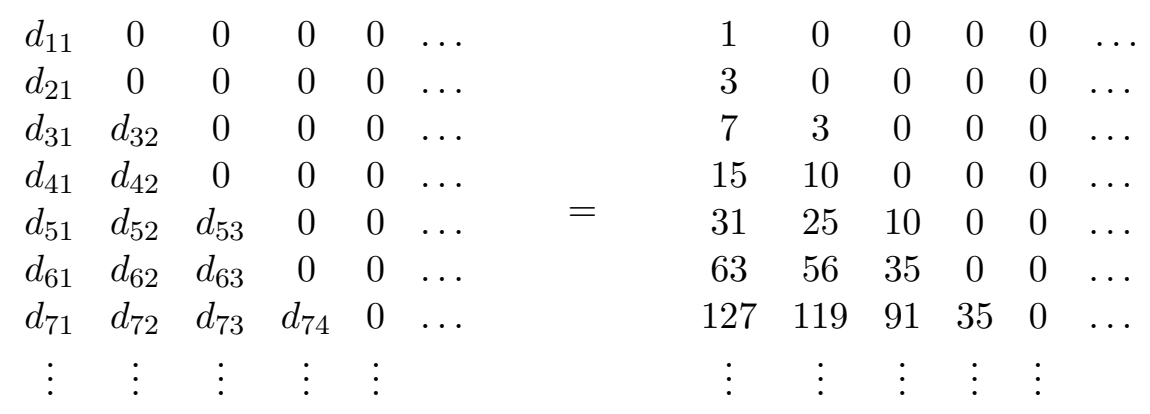

By Proposition [17, if $d_{r j}>0$, then we have

$$
d_{r j}=d_{r-1, j-1}+d_{r-1, j} .
$$

We have the following values of $\overline{\mathcal{T}}_{1}, \overline{\mathcal{T}}_{2}, \ldots, \overline{\mathcal{T}}_{7}$ :

$$
\begin{aligned}
& S_{22} \\
& 3 S_{34} \\
& 7 S_{46}+3 S_{55} \\
& 15 S_{58}+10 S_{67} \\
& 31 S_{6,10}+25 S_{79}+10 S_{88} \\
& 63 S_{7,12}+56 S_{8,11}+35 S_{9,10} \\
& 127 S_{8,14}+119 S_{9,13}+91 S_{10,12}+35 S_{11,11} .
\end{aligned}
$$

Summing up all our considerations, we get the main result of the present paper. It gives the desired Thom polynomial in a parametric form (the parameter being $r$ ).

Theorem 20 For $r \geq 1$, the Thom polynomial for $I_{2,2}(r)$ is equal to

$$
\sum_{i=0}^{r-1} \sum_{\{j \geq 1:} d_{i+2 j \leq r+1\}} S_{r-i, j} S_{i, r+j, 2 r-i-j+1},
$$

where the coefficients

$$
d_{r-i, j}=\langle j-1, r-i-j\rangle
$$

are given by Eq. (54) (or (55)). 
We have the following values of $\mathcal{T}_{1}, \mathcal{T}_{2}=\Phi\left(\mathcal{T}_{1}\right)+\overline{\mathcal{T}}_{2}, \ldots, \mathcal{T}_{7}=\Phi\left(\mathcal{T}_{6}\right)+\overline{\mathcal{T}}_{7}$ :

$$
\begin{aligned}
& S_{22} \\
& S_{133}+3 S_{34} \\
& S_{244}+3 S_{145}+7 S_{46}+3 S_{55} \\
& S_{355}+3 S_{256}+7 S_{157}+3 S_{166}+15 S_{58}+10 S_{67} \\
& S_{466}+3 S_{367}+7 S_{268}+3 S_{277}+15 S_{169}+10 S_{178}+31 S_{6,10}+25 S_{79}+10 S_{88} \\
& S_{577}+3 S_{478}+7 S_{379}+3 S_{388}+15 S_{2,7,10}+10 S_{289}+31 S_{1,7,11}+25 S_{1,8,10}+10 S_{199}+ \\
& 63 S_{7,12}+56 S_{8,11}+35 S_{9,10} \\
& S_{688}+3 S_{589}+7 S_{4,8,10}+3 S_{499}+15 S_{3,8,11}+10 S_{3,10,10}+31 S_{2,8,12}+25 S_{2,9,11}+ \\
& 10 S_{2,9,10}+63 S_{1,8,13}+56 S_{1,9,12}+35 S_{1,10,11}+127 S_{8,14}+119 S_{9,13}+91 S_{10,12}+35 S_{11,11} .
\end{aligned}
$$

Acknowledgments Though the author of the present paper is responsible for the exposition of the details, many computations here were done together with Alain Lascoux. This help is gratefully acknowledged. We also thank Richard Rimanyi for introducing us to his paper [31, Andrzej Weber for his assistance on singularities, and Ozer Ozturk for pointing out several defects of the manuscript.

Note After completion of the first version 26] of this paper, we received the preprint 8 containing a Chern monomial expression for the Thom series of $I_{2,2}$ supported by a computer evidence. In July 2006, we have received a letter from Kazarian [12] informing us that he has found another derivation of the Thom polynomial for $I_{2,2}$ based on geometric considerations.

\section{References}

[1] V. Arnold, V. Vasilev, V. Goryunov, O. Lyashko, Singularities. Local and global theory, Enc. Math. Sci. vol. 6 (Dynamical Systems VI), Springer, 1993.

[2] G. Berczi, L. Feher, R. Rimanyi, Expressions for resultants coming from the global theory of singularities, in: "Topics in algebraic and noncommutative geometry", (L. McEwan et al. eds.), Contemporary Math. AMS 324 (2003), $63-69$.

[3] A. Berele, A. Regev, Hook Young diagrams with applications to combinatorics and to representation theory of Lie superalgebras, Adv. in Math. 64 (1987), $118-175$.

[4] J. Damon, Thom polynomials for contact singularities, Ph.D. Thesis, Harvard, 1972 .

[5] A. Du Plessis, C.T.C. Wall, The geometry of topological stability, Oxford Math. Monographs, 1995. 
[6] L. Feher, B. Komuves, On second order Thom-Boardman singularities, Fund. Math. 191 (2006), 249-264.

[7] L. Feher, R. Rimanyi, Calculation of Thom polynomials and other cohomological obstructions for group actions, in: "Real and complex singularities (San Carlos 2002)" (T. Gaffney and M. Ruas eds.), Contemporary Math. 354, (2004), 69-93.

[8] L. Feher, R. Rimanyi, On the structure of Thom polynomials of singularities, Preprint (September 2005).

[9] W. Fulton, P. Pragacz, Schubert varieties and degeneracy loci, Springer LNM 1689 (1998).

[10] K. Jänich, Symmetry properties of singularities of $C^{\infty}$-functions, Math. Ann. 238 (1979), 147-156.

[11] M.E. Kazarian, Characteristic classes of singularity theory, in: "The ArnoldGelfand mathematical seminars: Geometry and singularity theory" (1997), $325-340$.

[12] M. E. Kazarian, Classifying spaces of singularities and Thom polynomials, in: "New developments in singularity theory", NATO Sci. Ser. II Math. Phys. Chem., 21, Kluwer Acad. Publ., Dordrecht (2001), 117-134.

[13] M. E. Kazarian, Letter to the author, dated July 4, 2006.

[14] S. Kleiman, The enumerative theory of singularities, in: "Real and complex singularities, Oslo 1976" (P. Holm ed.) (1978), 297-396.

[15] D. Laksov, A. Lascoux, A. Thorup, On Giambelli's theorem for complete correlations, Acta Math. 162 (1989), 143-199.

[16] A. Lascoux, Symmetric functions and combinatorial operators on polynomials, CBMS/AMS Lectures Notes 99, Providence (2003).

[17] A. Lascoux, Addition of \pm 1 : application to arithmetic, Séminaire Lotharingien de Combinatoire, B52a (2004), 9 pp.

[18] A. Lascoux, M-P. Schützenberger, Formulaire raisonné de fonctions symétriques, Université Paris 7, 1985.

[19] I.G. Macdonald, Symmetric functions and Hall-Littlewood polynomials, Oxford Math. Monographs, Second Edition, 1995.

[20] O. Ozturk, On Thom polynomials for $A_{4}(-)$ via Schur functions, Preprint, IMPAN Warszawa 2006 (670) - to appear in Serdica Math. J.

[21] I. Porteous, Simple singularities of maps, in: "Proc. Liverpool Singularities I", Springer LNM 192 (1971), 286-307.

[22] P. Pragacz, Note on elimination theory, Indagationes Math. 49 (1987), 215221. 
[23] P. Pragacz, Enumerative geometry of degeneracy loci, Ann. Sc. Ec. Norm. Sup. 21 (1988), 413-454.

[24] P. Pragacz, Algebro-geometric applications of Schur S- and Q-polynomials, in: "Topics in invariant theory" - Séminaire d'Algèbre Dubreil-Malliavin 19891990 (M-P. Malliavin ed.), Springer LNM 1478 (1991), 130-191.

[25] P. Pragacz Symmetric polynomials and divided differences in formulas of intersection theory, in: "Parameter spaces" (P. Pragacz ed.), Banach Center Publications 36 (1996), 125-177.

[26] P. Pragacz, Thom polynomials and Schur functions I, math.AG/0509234.

[27] P. Pragacz, Thom polynomials and Schur functions: towards the singularities $A_{i}(-)$, Preprint MPIM Bonn 2006 (139).

[28] P. Pragacz, Thom polynomials and Schur functions: the singularities $A_{3}(-)$, in preparation.

[29] P. Pragacz, A. Thorup, On a Jacobi-Trudi identity for supersymmetric polynomials, Adv. in Math. 95 (1992), 8-17.

[30] P. Pragacz, A. Weber, Positivity of Schur function expansions of Thom polynomials, Preprint, math.AG/0605308 and MPIM Bonn 2006 (60) - to appear in Fund. Math.

[31] R. Rimanyi, Thom polynomials, symmetries and incidences of singularities, Inv. Math. 143 (2001), 499-521.

[32] R. Rimanyi, A. Szücs, Generalized Pontrjagin-Thom construction for maps with singularities, Topology 37 (1998), 1177-1191.

[33] H. Schubert, Allgemeine Anzahlfunctionen für Kegelschnitte, Flächen und Raüme zweiten Grades in n Dimensionen, Math. Ann. 45 (1894), 153-206.

[34] J. Stembridge, A characterization of supersymmetric polynomials, J. of Algebra 95 (1985), 439-444.

[35] R. Thom, Les singularités des applications différentiables, Ann. Inst. Fourier 6 (1955-56), 43-87.

[36] C.T.C. Wall, A second note on symmetry of singularities, Bull. London Math. Soc. 12 (1980), 347-354. 